\title{
LAGRANGIAN AND HAMILTONIAN FUNCTIONS FOR RELATIVISTIC SYSTEM OF CHARGED PARTICLES
}

\author{
L. F. Blazhyjevskyj, B. V. Budnyj, Yu. S. Krynytskyj \\ Ivan Franko National University of Lviv, Department for Theoretical Physics \\ 12 Drahomanov Str., Lviv, UA-79005, Ukraine
}

(Received January 23, 2003; received in final form Juny 19, 2003)

\begin{abstract}
A relativistic generalization of Lagrangian and Hamiltonian functions for the classical system of charged particles is considered. Local Lagrangian expression in the first approximation by the coupling constant $e^{2}$ is obtained. This result is shown to be ambiguous. However, this ambiguity does not affect the considered approximation. Relativistic Hamiltonian function with screened interaction is found on applying some additional approximations.

Key words: relativistic statistical mechanics, quasistatic approximation, nonlocal Lagrangian, screened interaction.
\end{abstract}

PACS number(s): 05.20.-y, 03.30.+p, 03.50.De

\section{INTRODUCTION}

It is well known that a complete description of relativistic systems of interacting particles rests upon field theories. However, relativistic effects are considered to be small in many applications. If that is the case, one can restrict oneself to the post-Newtonian (or weakly relativistic) approximation, which implies us taking into account corrections of order $c^{-2}$ only ( $c$ is the speed of light in vacuum). The field degrees of freedom do not affect this approximation, therefore the state of the system can be described by particle variables only. This allows to develop the theory resting on Lagrangian (or Hamiltonian) function of the system of particles as in the nonrelativistic case. Well known examples are Darwin [1], EinsteinInfeld-Hoffman [2] and Kennedy [3] Lagrangians which describe charged, gravitating and neutral particles, respectively.

The post-Newtonian approximation is applicable when particle velocities are considerably smaller than the speed of light. In the present work this condition is not mandatory. At the same time, the radiation effects are neglected. An example of the system in which this can be realized is the optically thin relativistic plasma.

The paper is organized as follows. Section II gives some considerations on the action integral of the system of charged particles with direct interaction as well as expressions for the action in Lagrangian and canonical variables. In Section III relativistic generalization of
Lagrangian function of the system of charged particles is found using quasistatic approximation which is equivalent to the first order approximation by the coupling constant. This approximation gives the local Lagrangian expression which is ambiguous. The said ambiguity does not affect the considered approximation and various expressions for Lagrangian can therefore be considered as equivalent in first approximation by $e^{2}$. In order to obtain a Hamiltonian of the system of charged particles some approximations in addition to the first approximation by the coupling constant are applied in Section IV. The random phase approximation changes many-particle interactions to an effective screened pair interaction. The discussion of the Hamiltonian function and approximations validity is presented in the last section.

\section{ACTION INTEGRAL}

Let us consider a relativistic system of $N$ charged particles that is localized in a volume $V$. The complete action integral of such a system is a sum of the action $S_{\mathrm{f}}$ of the field produced by charges and action $S_{\mathrm{p}}$ of charges coupled to the field, that is:

$$
S=S_{\mathrm{p}}+S_{\mathrm{f}}
$$

In Lagrangian variables these are

$$
\begin{aligned}
& S_{\mathrm{f}}=\frac{1}{8 \pi} \int d t \int d \mathbf{r}\left[\left(-\nabla \varphi-\frac{1}{c} \frac{\partial \mathbf{A}}{\partial t}\right)^{2}-(\boldsymbol{\nabla} \times \mathbf{A})^{2}\right] \equiv \frac{1}{8 \pi} \int d t \int d \mathbf{r}\left(E^{2}-H^{2}\right), \\
& S_{\mathrm{p}}=S_{0}+S_{\mathrm{int}}=\int d t L_{0}+\int d t \int d \mathbf{r}\left(\frac{1}{c} \mathbf{j} \cdot \mathbf{A}-\rho \varphi\right),
\end{aligned}
$$

where $\mathbf{A}, \varphi$ and $\mathbf{E}, \mathbf{H}$ are electromagnetic potentials and fields; $\rho$ and $\mathbf{j}$ are charge and current densities, respectively: 


$$
\rho(\mathbf{r}, t)=\sum_{j} e_{j} \delta\left(\mathbf{r}-\mathbf{r}_{j}(t)\right), \quad \mathbf{j}(\mathbf{r}, t)=\sum_{j} e_{j} \mathbf{v}_{j}(t) \delta\left(\mathbf{r}-\mathbf{r}_{j}(t)\right)
$$

$L_{0}$ is the free particles relativistic Lagrangian:

$$
L_{0}=-\sum_{j} m_{j} c^{2}\left(1-v_{j}^{2} / c^{2}\right)^{1 / 2}
$$

It is convenient to modify the field portion of the action. If one integrates by parts in (2), then $S_{\mathrm{f}}$ can be represented in the form

$$
S_{\mathrm{f}}=\frac{1}{8 \pi} \int d t \int d \mathbf{r}\left\{-\varphi\left(\Delta \varphi+\frac{1}{c} \frac{\partial}{\partial t} \boldsymbol{\nabla} \cdot \mathbf{A}\right)+\mathbf{A} \cdot\left[\Delta \mathbf{A}-\frac{1}{c^{2}} \frac{\partial^{2} \mathbf{A}}{\partial t^{2}}-\boldsymbol{\nabla}\left(\boldsymbol{\nabla} \cdot \mathbf{A}+\frac{1}{c} \frac{\partial \varphi}{\partial t}\right)\right]\right\}
$$

The representations (2) and (6) are equivalent. Indeed, one can easily make certain that from the variational principle $\delta\left(S_{\mathrm{f}}+S_{\mathrm{int}}\right)=0$ where $S_{\mathrm{f}}$ is determined either by (2) or (6) one can obtain the field equations

$$
\begin{aligned}
& \Delta \mathbf{A}-\frac{1}{c^{2}} \frac{\partial^{2} \mathbf{A}}{\partial t^{2}}-\boldsymbol{\nabla}\left(\boldsymbol{\nabla} \cdot \mathbf{A}+\frac{1}{c} \frac{\partial \varphi}{\partial t}\right)=-\frac{4 \pi}{c} \mathbf{j}, \\
& \Delta \varphi+\frac{1}{c} \frac{\partial}{\partial t} \boldsymbol{\nabla} \cdot \mathbf{A}=-4 \pi \rho .
\end{aligned}
$$

Field variables are to be replaced by the solutions of field equations (7) in order to proceed to the theory with direct interaction between particles. Then from (6), (7) one gets

$$
S_{\mathrm{f}}=\frac{1}{2} \int d t \int d \mathbf{r}\left(\rho \varphi-\frac{1}{c} \mathbf{j} \cdot \mathbf{A}\right),
$$

that is $S_{\mathrm{int}}=-2 S_{\mathrm{f}}$ when the field degrees of freedom are frozen out. This equality was also abtained, for example, in [4-6] as an approximate result, so it is worth emphasizing that (9) is an exact relation in the theory of direct interparticle action.

From (1), (3), (9) the action integral of a relativistic system of charged particles within the framework of direct interaction can be written as

$$
S=\int d t\left(L_{0}+L_{\mathrm{int}}\right)
$$

where we have introduced the notation

$$
\begin{aligned}
L_{\mathrm{int}} & =\frac{1}{2} \int d \mathbf{r}\left(\frac{1}{c} \mathbf{j} \cdot \mathbf{A}-\rho \varphi\right) \\
& =\frac{1}{2} \sum_{j} e_{j}\left[\frac{1}{c} \mathbf{v}_{j}(t) \cdot \mathbf{A}\left(\mathbf{r}_{j}(t), t\right)-\varphi\left(\mathbf{r}_{j}(t), t\right)\right] .
\end{aligned}
$$

One can see from the stated above that $L_{\text {int }}$ can be ex- pressed via fields as well:

$$
L_{\mathrm{int}}=\frac{1}{8 \pi} \int d \mathbf{r}\left(H^{2}-E^{2}\right)
$$

It is clear that the field values in (10)-(12) are no longer independent variables.

Let us consider the same in canonical variables. The action integral of particles in the external field is then described by the formulae

$$
\begin{aligned}
& S_{\mathrm{p}}=\int d t \sum_{j}\left\{\mathbf{p}_{j} \cdot \mathbf{v}_{j}-\left[\varepsilon\left(\pi_{j}\right)+e_{j} \varphi\left(\mathbf{r}_{j}, t\right)\right]\right\}, \\
& \varepsilon\left(\pi_{j}\right)=\left(m_{j}^{2} c^{4}+c^{2} \pi_{j}^{2}\right)^{1 / 2}, \\
& \boldsymbol{\pi}_{j}=\mathbf{p}_{j}-\frac{e_{j}}{c} \mathbf{A}\left(\mathbf{r}_{j}, t\right) .
\end{aligned}
$$

The field part of the action is still determined by formula (9) with particle velocities $\mathbf{v}_{j}$ (in formula (4) for the current density $\mathbf{j}$ ) expressed via conjugate momenta, that is

$$
\mathbf{v}_{j}=\frac{c^{2} \boldsymbol{\pi}_{j}}{\varepsilon\left(\pi_{j}\right)} .
$$

Thus, integral (10) in canonical variables will read

$$
S=\int d t\left[\sum_{j} \mathbf{p}_{j} \cdot \mathbf{v}_{j}-H\right],
$$

where we have introduced the notation

$$
\begin{aligned}
H & =\sum_{j}\left[\varepsilon\left(\pi_{j}\right)+\frac{c e_{j}}{2 \varepsilon\left(\pi_{j}\right)} \boldsymbol{\pi}_{j}\right. \\
& \left.\times \mathbf{A}\left(\mathbf{r}_{j}(t), t\right)+\frac{1}{2} e_{j} \varphi\left(\mathbf{r}_{j}(t), t\right)\right] .
\end{aligned}
$$


Expressions (10), (16) will make sense of the action integral describing the system of charged particles with direct interaction if one inserts the solutions of potential equations into (11), (17). Generally the action found in this way will be nonlocal, i. e., $S\left(t_{0}, t\right) \neq S\left(t_{0}, t^{\prime}\right)+$ $S\left(t^{\prime}, t\right),\left(t_{0}<t^{\prime}<t\right)$. The local action can be obtained (and therefore (11), (17) can be identified with usual Lagrangian or Hamiltonian functions) approximately only.

\section{LAGRANGIAN FUNCTION}

In order to obtain the Lagrangian let us consider potentials $\varphi$ and $\mathbf{A}$. It is convenient to use the Coulomb gauge $\boldsymbol{\nabla} \cdot \mathbf{A}=0$ and work with Fourier transforms:

$$
\begin{aligned}
\Phi(\mathbf{r}, t) & =\frac{1}{V} \sum_{\mathbf{k}} \Phi_{\mathbf{k}}(t) e^{i \mathbf{k} \cdot \mathbf{r}} \\
\Phi_{\mathbf{k}}(t) & =\int d \mathbf{r} \Phi(\mathbf{r}, t) e^{-i \mathbf{k} \cdot \mathbf{r}} .
\end{aligned}
$$

The fourier transform $\varphi_{\mathbf{k}}(t)$ of the scalar potential is then defined by the formula

$$
\varphi_{\mathbf{k}}(t)=\frac{4 \pi}{k^{2}} \rho_{\mathbf{k}}(t)=\frac{4 \pi}{k^{2}} \sum_{j} e_{j} e^{-i \mathbf{k} \cdot \mathbf{r}_{j}} .
$$

The vector potential will satisfy the equation

$$
\left(k^{2}+\frac{1}{c^{2}} \frac{d^{2}}{d t^{2}}\right) \mathbf{A}_{\mathbf{k}}(t)=\frac{4 \pi}{c} \mathbf{j}_{\mathbf{k}}^{\perp}(t)
$$

where

$$
\mathbf{j}_{\mathbf{k}}^{\perp}(t)=\sum_{j} e_{j} e^{-i \mathbf{k} \cdot \mathbf{r}_{j}}\left(\mathbf{v}_{j}-\hat{\mathbf{k}}\left(\hat{\mathbf{k}} \cdot \mathbf{v}_{j}\right)\right)
$$

is the transversal current (hat over vector denotes a unitary vector both here and in what follows). One can remove the second time derivative from (19) in the static approximation. Then

$$
\mathbf{A}_{\mathbf{k}}(t)=\frac{4 \pi}{c k^{2}} \mathbf{j}_{\mathbf{k}}^{\perp}(t)
$$

Turning to Fourier representation in (11) and inserting the static potentials (18), (21) in place of $\mathbf{A}_{\mathbf{k}}, \varphi_{\mathbf{k}}$ one finds after exclusion of the self-action terms:

$$
\begin{aligned}
L_{\mathrm{int}} & =-\frac{1}{2 V} \sum_{\mathbf{k}} \frac{4 \pi}{k^{2}} \sum_{j \neq l} e_{j} e_{l} e^{i \mathbf{k} \cdot \mathbf{r}_{j l}} \\
& \times\left\{1-\frac{1}{c^{2}}\left[\mathbf{v}_{j} \cdot \mathbf{v}_{l}-\left(\hat{\mathbf{k}} \cdot \mathbf{v}_{j}\right)\left(\hat{\mathbf{k}} \cdot \mathbf{v}_{l}\right)\right]\right\} .
\end{aligned}
$$

In coordinate representation (22) will read

$$
\begin{aligned}
L_{\mathrm{int}} & =-\frac{1}{2} \sum_{j \neq l} \frac{e_{j} e_{l}}{r_{j l}}\left\{1-\frac{1}{2 c^{2}}\left[\mathbf{v}_{j} \cdot \mathbf{v}_{l}\right.\right. \\
& \left.\left.-\left(\mathbf{v}_{j} \cdot \hat{\mathbf{r}}_{j l}\right)\left(\mathbf{v}_{l} \cdot \hat{\mathbf{r}}_{j l}\right)\right]\right\} .
\end{aligned}
$$

This is a well-known Darwin interaction Lagrangian which describes the post-Newtonian system of charged particles.

It seems natural to consider the possibility of a relativistic generalization of Lagrangian (22) when the weakly relativistic condition $v \ll c$ does not apply. One can proceed from formula (12) after rewriting it in the form

$$
L_{\mathrm{int}}=\frac{1}{8 \pi V} \sum_{\mathbf{k}}\left(\mathbf{H}_{\mathbf{k}} \cdot \mathbf{H}_{-\mathbf{k}}-\mathbf{E}_{\mathbf{k}} \cdot \mathbf{E}_{-\mathbf{k}}\right)
$$

For the purpose of generalization one can use in (24) the quasistatic solutions of the field equations instead of static ones. The quasistatic fields, i. e. fields of uniformly moving charges, are given by formulae [4]

$$
\begin{aligned}
\mathbf{E}_{\mathbf{k}} & =\frac{4 \pi i}{k^{2}} \sum_{j} e_{j} e^{-i \mathbf{k} \cdot \mathbf{r}_{j}} \frac{-\mathbf{k}+\mathbf{v}_{j}\left(\mathbf{k} \cdot \mathbf{v}_{j}\right) / c^{2}}{s_{j}}, \\
\mathbf{H}_{\mathbf{k}} & =\frac{4 \pi i}{c k^{2}} \sum_{j} e_{j} e^{-i \mathbf{k} \cdot \mathbf{r}_{j}} \frac{\mathbf{k} \times \mathbf{v}_{j}}{s_{j}}, \\
s_{j} & =1-\left(\hat{\mathbf{k}} \cdot \mathbf{v}_{j}\right)^{2} / c^{2} .
\end{aligned}
$$

Upon using these expressions in (24) one obtains

$$
\begin{aligned}
L_{\mathrm{int}} & =-\frac{1}{2 V} \sum_{\mathbf{k}} \frac{4 \pi}{k^{2}} \sum_{j \neq l} e_{j} e_{l} e^{i \mathbf{k} \cdot \mathbf{r}_{j l}} \\
& \times\left\{1-\frac{1}{c^{2} s_{j} s_{l}}\left[\mathbf{v}_{j} \cdot \mathbf{v}_{l}-\left(\hat{\mathbf{k}} \cdot \mathbf{v}_{j}\right)\left(\hat{\mathbf{k}} \cdot \mathbf{v}_{l}\right)\right]\right. \\
& \left.\times\left[1-\frac{1}{c^{2}}\left(\hat{\mathbf{k}} \cdot \mathbf{v}_{j}\right)\left(\hat{\mathbf{k}} \cdot \mathbf{v}_{l}\right)\right]\right\} .
\end{aligned}
$$

The sum $L_{0}+L_{\text {int }}$ ( $L_{0}$ being expressed by (5)) can be treated as the model Lagrangian that approximately describes the relativistic system of charged particles at any velocities. A similar Lagrangian was previously found in [5] basing on formula (11) and is different from expression (25). This is explained by the following. The exact direct interaction Lagrangian is obtained after substituting full solutions of the field equations into (11) or (12) and is nonlocal. These nonlocal Lagrangians found from either (11) or (12) are equivalent up to a total derivative. On the other hand, inserting the quasistatic fields results in local expression. The same can be achieved by neglecting higher derivative terms ( $\ddot{\mathbf{r}}$ and higher) in the higher 
derivative expansion of the nonlocal Lagrangian. After excluding higher derivatives one gets a different outcome from formulae (11) and (12). Moreover, one can generate infinite number of approximate Lagrangians like (25) by inserting potentials in a certain gauge into (11) and excluding higher derivatives. Nevertheless, it can be shown that these expressions are valid in first approximation by the coupling constant $e^{2}$ and are equivalent in this approximation.

Cosider the nonlocal Lagrangian which describes the system of charged particles after field degrees of freedom having been excluded. This Lagrangian can be written as higher derivative expansion (which was first done by Kerner [7]):

$$
L=L_{0}+e^{2}\left(L_{1}+L_{2}\right),
$$

where $L_{0}$ is given by (5), $L_{1}$ is the part of interaction Lagrangian that depends on particle positions and velocities only, $L_{2}$ contains higher derivative terms. It turns out that $L_{2}$ can always be written as

$$
L_{2}=\sum_{j} \ddot{\mathbf{r}}_{j} \cdot \boldsymbol{\Phi}_{j}
$$

where $\boldsymbol{\Phi}_{j}$ is a function of particle coordinates, velocities and higher derivatives. This term then can be excluded from Lagrangian in the first order by $e^{2}$ on applying certain coordinate transformation (see [8] and references therein for a detailed discussion). Namely, let us make the following coordinate transformation:

$$
\mathbf{r}_{j} \rightarrow \mathbf{r}_{j}+e^{2} \mathbf{X}_{j}
$$

Upon expanding by $e^{2}$ (26) will read

$$
\begin{aligned}
L & =L_{0}+e^{2}\left(L_{1}+\sum_{j} \frac{\partial L_{0}}{\partial \mathbf{v}_{j}} \cdot \dot{\mathbf{X}}_{j}\right. \\
& \left.+\sum_{j} \ddot{\mathbf{r}}_{j} \cdot \boldsymbol{\Phi}_{j}\right)+\mathcal{O}\left(e^{4}\right) .
\end{aligned}
$$

This can be rewritten after the total derivative having been dropped:

$$
\begin{aligned}
L & =L_{0}+e^{2}\left(L_{1}-\sum_{j} \frac{d}{d t}\left(\frac{\partial L_{0}}{\partial \mathbf{v}_{j}}\right) \cdot \mathbf{X}_{j}\right. \\
& \left.+\sum_{j} \ddot{\mathbf{r}}_{j} \cdot \boldsymbol{\Phi}_{j}\right)+\mathcal{O}\left(e^{4}\right) .
\end{aligned}
$$

One can now choose $\mathbf{X}_{j}$ to satisfy the condition

$$
\frac{d}{d t}\left(\frac{\partial L_{0}}{\partial \mathbf{v}_{j}}\right) \cdot \mathbf{X}_{j}=\ddot{\mathbf{r}}_{j} \cdot \boldsymbol{\Phi}_{j}
$$

that is

$$
\frac{m_{j}}{\sqrt{1-v_{j}^{2} / c^{2}}}\left[\mathbf{X}_{j}+\frac{\mathbf{v}_{j}\left(\mathbf{v}_{j} \cdot \mathbf{X}_{j}\right)}{c^{2}\left(1-v_{j}^{2} / c^{2}\right)}\right]=\mathbf{\Phi}_{j},
$$

from where

$$
\mathbf{X}_{j}=\frac{\sqrt{1-v_{j}^{2} / c^{2}}}{m_{j}}\left[\boldsymbol{\Phi}_{j}-\frac{\mathbf{v}_{j}}{c^{2}}\left(\boldsymbol{\Phi}_{j} \cdot \mathbf{v}_{j}\right)\right] .
$$

Thus, after making transformation (28) with $\mathbf{X}_{j}$ of (33) the Lagrangian (26) will read

$$
L=L_{0}+e^{2} L_{1}+\mathcal{O}\left(e^{4}\right),
$$

that is, higher derivative terms can be omitted in the first order by $e^{2}$.

Consider now $L^{\prime}$ being another nonlocal Lagrangian obtained as described above. Obviously, $L$ and $L^{\prime}$ differ by the total derivative of a function depending on coordinates and generally derivatives of any order of the time coordinates:

$$
\begin{aligned}
L^{\prime} & =L_{0}+e^{2}\left(L_{1}^{\prime}+L_{2}^{\prime}\right), \\
L & =L^{\prime}+e^{2} \frac{d}{d t} F\left(\mathbf{r}, \frac{d^{n} \mathbf{r}}{d t^{n}}\right),
\end{aligned}
$$

$L_{1}^{\prime}$ and $L_{2}^{\prime}$ having the same sense as in (26). It is easy to see that the local part of Lagrangian (26) $L_{\mathrm{loc}} \equiv$ $L_{0}+e^{2} L_{1}$ is equivalent up to the total derivative $e^{2} d F / d t$ to the following expression

$$
L_{0}+e^{2}\left(L_{1}^{\prime}-L_{2}^{*}\right)
$$

where $L_{2}^{*}$ contains higher derivatives from $e^{2} d F / d t$. On applying the corresponding coordinate transformation (28) the latter formula reads

$$
L_{0}+e^{2} L_{1}^{\prime}+\mathcal{O}\left(e^{4}\right) \equiv L_{\mathrm{loc}}^{\prime}+\mathcal{O}\left(e^{4}\right)
$$

Therefore, Lagrangians $L_{\mathrm{loc}}$ and $L_{\mathrm{loc}}^{\prime}$ can be regarded as equivalent in the first approximation by the coupling constant $e^{2}$. 


\section{HAMILTONIAN FUNCTION}

Now we are going to make the similar calculations in canonical variables. Although a vector potential is still described by expressions (19), (20), particle velocities $\mathbf{v}_{j}$ in (20) have to be reformulated in terms of conjugate momenta according to (15). So we obtain a complicated nonlinear equation for $\mathbf{A}_{\mathbf{k}}(t)$. In order to solve it some simplifications need to be applied.

1. Linearization. Let us expand $\varepsilon(\pi)$ in (15) into Taylor series by the powers of charge value. In the linear approximation we will have:

$$
\begin{aligned}
& \mathbf{v}_{j}=\frac{c^{2} \mathbf{p}_{j}}{\varepsilon_{j}}-\frac{c e_{j}}{\varepsilon_{j}}\left[\mathbf{A}\left(\mathbf{r}_{j}\right)-\frac{c^{2} \mathbf{p}_{j}}{\varepsilon_{j}^{2}}\left(\mathbf{A}\left(\mathbf{r}_{j}\right) \cdot \mathbf{p}_{j}\right)\right] \\
& \varepsilon_{j}^{2} \equiv m_{j}^{2} c^{4}+c^{2} p_{j}^{2}
\end{aligned}
$$

The last term of (37) can be averaged by momentum orientations writing $p_{j}^{\mu} p_{j}^{\nu}=\frac{1}{3} p_{j}^{2} \delta_{\mu \nu}\left(\mu, \nu=[x, y, z], \delta_{\mu \nu}\right.$ is Kronecker delta). Representing $\mathbf{A}\left(\mathbf{r}_{j}\right)$ by its Fourier expansion one can see that the transversal current (20) will read

$$
\begin{aligned}
\mathbf{j}_{\mathbf{k}}^{\perp} & =\sum_{j} \frac{c^{2} e_{j}}{\varepsilon_{j}} e^{-i \mathbf{k} \cdot \mathbf{r}_{j}}\left[\mathbf{p}_{j}-\hat{\mathbf{k}}\left(\hat{\mathbf{k}} \cdot \mathbf{p}_{j}\right)\right] \\
& -\sum_{j} \frac{c e_{j}^{2}}{\varepsilon_{j}}\left(1-\frac{c^{2} p_{j}^{2}}{3 \varepsilon_{j}^{2}}\right) \\
& \times \frac{1}{V} \sum_{\mathbf{q}}\left[\mathbf{A}_{\mathbf{q}}-\hat{\mathbf{k}}\left(\hat{\mathbf{k}} \cdot \mathbf{A}_{\mathbf{q}}\right)\right] e^{i(\mathbf{q}-\mathbf{k}) \cdot \mathbf{r}_{j}} \\
& \equiv \mathbf{j}_{\mathbf{k}}^{(0)}-\mathbf{j}_{\mathbf{k}}^{(1)}(\mathbf{A})
\end{aligned}
$$

Upon inserting this into equation for $\mathbf{A}_{\mathbf{k}}$ one finds the vector potential as a function of particle coordinates and momenta. We will use the simplest static expression (21). Then the equation for $\mathbf{A}_{\mathbf{k}}$ will be the following:

$$
k^{2} \mathbf{A}_{\mathbf{k}}=\frac{4 \pi}{c}\left[\mathbf{j}_{\mathbf{k}}^{(0)}+\mathbf{j}_{\mathbf{k}}^{(1)}(\mathbf{A})\right] .
$$

It is also necessary to linearize the function $H$. On expanding (17) by the powers of $e_{j}$ it will read

$$
\begin{aligned}
H & =\sum_{j} \varepsilon_{j}+\frac{1}{2 V} \sum_{\mathbf{k}} \sum_{j} e_{j} e^{i \mathbf{k} \cdot \mathbf{r}_{j}}\left(\varphi_{k}-\frac{c}{\varepsilon_{j}}\left(\mathbf{p}_{j} \cdot \mathbf{A}_{\mathbf{k}}\right)\right) \\
& +\mathcal{O}\left(e_{j}^{3}\right)
\end{aligned}
$$

Note that quadratic in $e_{j}$ terms in (17) are reciprocally cancelled.

2. One may apply the random phase approximation which means that the only term considered in $\mathbf{j}_{\mathbf{k}}^{(1)}(\mathbf{A})$ is the one with $\mathbf{q}=\mathbf{k}$. It is easy to see now that $\mathbf{j}_{\mathbf{k}}^{(1)}=\varkappa^{2} \mathbf{A}_{\mathbf{k}}$, where

$$
\varkappa^{2}=\sum_{j} \frac{4 \pi e_{j}^{2}}{V \varepsilon_{j}}\left(1-\frac{c^{2} p_{j}^{2}}{3 \varepsilon_{j}^{2}}\right)
$$

So the vector potential in canonical variables in the static case is given by

$$
\mathbf{A}_{\mathbf{k}}=\frac{4 \pi c}{k^{2}+\varkappa^{2}} \sum_{j} \frac{e_{j}}{\varepsilon_{j}} e^{-i \mathbf{k} \cdot \mathbf{r}_{j}}\left(\mathbf{p}_{j}-\hat{\mathbf{k}}\left(\hat{\mathbf{k}} \cdot \mathbf{p}_{j}\right)\right)
$$

To obtain the Hamiltonian function of the system of directly interacting particles it is now enough to apply expressions (18), (43) in (41) and exclude divergent selfenergy terms. The result can be written as

$$
H=\sum_{j} \varepsilon_{j}+\frac{1}{2} \sum_{j \neq l}\left(H_{j l}^{\mathrm{C}}+H_{j l}^{\mathrm{R}}\right)+\sum_{j}\left(\frac{1}{2} H_{j j}^{\mathrm{C}}+\frac{1}{2} H_{j j}^{\mathrm{R}}-U_{j}\right)
$$

where

$$
H_{j l}^{\mathrm{C}}=\frac{1}{V} \sum_{\mathbf{k}} \frac{4 \pi e_{j} e_{l}}{k^{2}} e^{i \mathbf{k} \cdot \mathbf{r}_{j l}}
$$

being the Coulomb interaction energy,

$$
H_{j l}^{\mathrm{R}}=-\frac{1}{V} \sum_{\mathbf{k}} \frac{4 \pi e_{j} e_{l}}{k^{2}+\varkappa^{2}} e^{i \mathbf{k} \cdot \mathbf{r}_{j l}} \frac{c^{2}}{\varepsilon_{j} \varepsilon_{l}}\left[\left(\mathbf{p}_{j} \cdot \mathbf{p}_{l}\right)-\left(\hat{\mathbf{k}} \cdot \mathbf{p}_{j}\right)\left(\hat{\mathbf{k}} \cdot \mathbf{p}_{l}\right)\right]
$$

is the energy of relativistic interactions. $U_{j}$ is the self-energy of a charged particle. In the considered approximation 
it is determined by the formula:

$$
U_{j}=\frac{1}{2 V} \sum_{\mathbf{k}} \frac{4 \pi e_{j}^{2}}{k^{2}}\left[1-\frac{c^{2}}{\varepsilon_{j}^{2}}\left(p_{j}^{2}-\left(\hat{\mathbf{k}} \cdot \mathbf{p}_{j}\right)^{2}\right)\right]
$$

It is easy to see that

$$
\frac{1}{2}\left(H_{j j}^{\mathrm{C}}+H_{j j}^{\mathrm{R}}\right)-U_{j}=\frac{2 \pi e_{j}^{2} c^{2}}{\varepsilon_{j}^{2} V} \sum_{\mathbf{k}}\left[\frac{1}{k^{2}}-\frac{1}{k^{2}+\varkappa^{2}}\right]\left[p_{j}^{2}-\left(\hat{\mathbf{k}} \cdot \mathbf{p}_{j}\right)^{2}\right]=\frac{1}{3} \varkappa e_{j}^{2} \frac{c^{2} p_{j}^{2}}{\varepsilon_{j}^{2}}
$$

Therefore, the last sum of (44) is nonzero. So the selfenergy exclusion in Hamiltonian variables is no longer reduced to the trivial neglect of $j=l$ terms. Since $H_{j j}^{\mathrm{R}}$ depends on the $\varkappa$ parameter it is affected by the number of particles in the system. Each particle's self-energy at the same time must not depend on their total amount. This is expression (48) which determines the difference between those energies. After joining this contribution and the first term in (44) we can write:

$$
H=\sum_{j} H_{j}^{*}+\frac{1}{2} \sum_{j \neq l}\left(H_{j l}^{\mathrm{C}}+H_{j l}^{\mathrm{R}}\right)
$$

where

$$
H_{j}^{*}=\varepsilon_{j}+\frac{1}{3} \varkappa e_{j}^{2} \frac{c^{2} p_{j}^{2}}{\varepsilon_{j}^{2}}
$$

and $H_{j l}^{\mathrm{C}}, H_{j l}^{\mathrm{R}}$ are expressed by formulae (45), (46).

\section{DISCUSSION}

Formula (49) can be regarded as relativistic generalization of the weakly relativistic Hamiltonian. It is complicated by the "screening radius" $\varkappa^{-1}$ dependence on momenta of all particles. In applications, however, one can replace $\varkappa^{2}$ by a certain mean value using for example the free particles distribution function. Then $\varkappa$ makes sense of some macroscopic parameter. Performing the sum over $\mathbf{k}$ in (46) one finds that

$$
H_{j l}^{\mathrm{R}}=-\frac{e_{j} e_{l} c^{2}}{r_{j l}} \frac{p_{j}^{\mu} p_{l}^{\nu}}{\varepsilon_{j} \varepsilon_{l}} \Phi_{\mu \nu}\left(\varkappa \mathbf{r}_{j l}\right)
$$

where

$$
\begin{aligned}
\Phi_{\mu \nu}(\mathbf{x}) & =\left(\delta_{\mu \nu}-\hat{x}_{\mu} \hat{x}_{\nu}\right) e^{-x} \\
& +\left(\delta_{\mu \nu}-3 \hat{x}_{\mu} \hat{x}_{\nu}\right)\left(\frac{e^{-x}}{x}+\frac{e^{-x}}{x^{2}}-\frac{1}{x^{2}}\right) .
\end{aligned}
$$

As one can see, there is the term $p_{j}^{\mu} p_{l}^{\nu}\left(\delta_{\mu \nu}-\hat{r}_{\mu} \hat{r}_{\nu}\right) / r_{j l}^{3}$ in $H_{j l}^{\mathrm{R}}$ in addition to the terms with the screening exponent. This term which is typical for dipole interaction dissapears after averaging by $\mathbf{r}_{j l}$ directions, i. e. the effective relativistic interaction is screened on average only.

Let us make some notes on the applied approximations. Lagrangian and Hamiltonian functions obtained in previous sections are valid in the first approximation by the coupling constant, which allows the exclusion of higher derivatives from the theory. From quantum electrodynamics it is known that radiation effects have a higher order of $e^{2}$ than elastic scattering, so in the same approximation one can neglect self-energy terms as well. This approximation is expected to be acceptable, for example, for optically thin plasma and breaks in the region $T \gtrsim m c^{2}$, where radiation effects cannot be omitted. It is necessary to note that there are other ways of dealing whith complications due to retarded electromagnetic interaction, one of which is introducing grassman valued particle charges $[9,10]$.

One may ask whether it is correct in the first approximation by $e^{2}$ to have the screening denominator dependent on the coupling constant. In this connection it is necessary to clarify that approximation by the coupling constant applies here to the direct interaction between two particles. The statistical many-particle effect of screening must be concidered in order to avoid divergencies due to a long range character of the interaction (see, e. g., [11-13]). An indirect accounting of many-particle interactions results in relativistic twoparticle interaction screening and the free particle energy renormalization in Hamiltonian function. Indeed equation (40) can be solved by successive approximations taking $\mathbf{j}_{\mathbf{k}}^{(1)}(\mathbf{A})$ as perturbation. It is clear that after inserting the solution found by such a method into (44) one obtains series for the relativistic interaction. Moreover, the $n$-th series member will contain $n+1$ sums over particle indices, that is, the relativistic interaction has a many-particle character [14]. These many-particle interactions can be replaced by effective pair interaction in the random phase approximation when only interactions with the same transmitted momentum are considered. This results in the geometric progression which leads to the screening denominator in (46). Relativistic interac- 
tions screening is a typical property of statistical systems. Classical and quantum mechanical problems usually imply the particle number $N$ to be small. Moreover, $N$ and $V$ are not connected in any way. That is why $\varkappa \rightarrow 0$ when $V \rightarrow \infty$ and expression (49) is significantly simplified. Since $\Phi_{\mu \nu}(\mathbf{x}) \underset{x \rightarrow 0}{\longrightarrow}\left(\delta_{\mu \nu}+\hat{x}_{\mu} \hat{x}_{\nu}\right) / 2$ it is easy to make sertain that Hamiltonian will read

$$
H=\sum_{j} \varepsilon_{j}+\frac{1}{2} \sum_{j \neq l} \frac{e_{j} e_{l}}{r_{j l}}-\frac{1}{2} \sum_{j \neq l} \frac{e_{j} e_{l}}{r_{j l}} \frac{c^{2}\left[\mathbf{p}_{j} \cdot \mathbf{p}_{l}+\left(\hat{\mathbf{r}}_{j l} \cdot \mathbf{p}_{j}\right)\left(\hat{\mathbf{r}}_{j l} \cdot \mathbf{p}_{l}\right)\right]}{2 \varepsilon_{j} \varepsilon_{l}}
$$

In a weakly relativistic approximation one obtains from the last formula familiar Darwin Hamiltonian. In statistical mechanics one has to perform the thermodynamic limit $N \rightarrow \infty, V \rightarrow \infty, N / V=$ const so $\varkappa^{2} \neq 0$. Evidently, the calculation of macroscopic properties has to be based on the Hamiltonian (49) or its weakly relativistic approximation. In the weakly relativistic approximation obtained relations are significantly simplified. If that is the case then

$$
\varkappa^{2} \simeq \sum_{j} 4 \pi e_{j}^{2} / m_{j} c^{2} \equiv \varkappa_{w}^{2}
$$

So the one-particle Hamiltonian (50) can be represented in the form

$$
\begin{aligned}
& H_{j}^{*}=m_{j} c^{2}+\frac{p_{j}^{2}}{2 m_{j}^{*}}-\frac{p_{j}^{4}}{8 m_{j}^{3} c^{2}}, \\
& m_{j}^{*}=m_{j}\left(1-\frac{2 e_{j}^{2} \varkappa_{w}}{3 m_{j} c^{2}}\right),
\end{aligned}
$$

and it is enough to replace the particle energy $\varepsilon_{j}$ in (46) by the rest energy. Such an approximate weakly relativistic Hamiltonian was obtained by one of the authors in [15] using the Legendre transformation. The same relations were found in [16] by means of canonical Bogoliubov's $u$-v-transformation. Similar results can also be received after taking into account the spins of particles and for the system of neutral atoms [13]. Basing on the field theory a weakly relativistic Hamiltonian function with the screened interaction of currents was obtained in [17] as well (however, the relativistic contributions responsible for the mass renormalization of (54) were not taken into account). A weakly relativistic approximation of Hamiltonian (49) was constructed in [18] resting on the field approach. The field variables exclusion method used in [18] is different from that of [17]. The screening effect in the nonrelativistic magnetic interaction Hamiltonian was considered in $[6,19]$ with some applications having been discussed. It is worth mentioning that the screening of magnetic interactions in the Hamiltonian of the system of charged particles for the first time was pointed out by Bohm and Pines in [20].
[1] C. G. Darwin, Philos. Mag. 39, 537 (1920).

[2] A. Einstein, L. Infeld, B. Hoffman, Ann. Math. 39, 65 (1938).

[3] F. D. Kennedy, Am. J. Phys. 40, 63 (1972).

[4] L. D. Landau, E. M. Lifschitz, Classical Theory of Fields (Pergamon, New York, 1972).

[5] B. A. Trubnikov, V. V. Kosachev, Zh. Eksp. Teor. Fiz. 66, 1311 (1974).

[6] H. Essén, J. Phys. A 32, 2297 (1999).

[7] E. J. Kerner, J. Math. Phys. 3, 35 (1962).

[8] C. Grosse-Knetter, Phys. Rev. D 49, 6709 (1994).

[9] H. Crater, L. Lusanna, Ann. Phys. 289, 87 (2001).

[10] D. Alba, H. Crater, L. Lusanna, Int. J. Mod. Phys. A 16, 3365 (2001).

[11] B. A. Trubnikov, V. V. Kosachev, Zh. Eksp. Teor. Fiz. 54, 939 (1968).
[12] B. A. Trubnikov, V. V. Kosachev, Nucl. Fusion 9, 53 (1969).

[13] L. F. Blazhievsky, H. B. Hil', S. S. Semak, J. Phys. Stud. 1, 1 (1996).

[14] R. P. Haida, Fiz. Elem. Chastits At. Yadra 13, 427 (1982).

[15] L. F. Blazhievsky, Ukr. Fiz. Zh. 20, 1273 (1975).

[16] L. F. Blazhyevskii, Yu. Krynytskyi, Condens. Matter Phys. 1, 569 (1998).

[17] R. D. Jones, A. Pytte, Phys. Fluids 23, 269 (1980).

[18] L. F. Blazhievskyi, H. B. Hil', V. S. Yanishevskii, Voprosy kvantovojteorii kondensirovannykh sred (Problems of the quantum theory of condensed matter) (Stiinza, Kishinev, 1990).

[19] H. Essén, Phys. Rev. E 56, 5858 (1997).

[20] D. Bohm, P. Pines, Phys. Rev. 82, 625 (1951). 


\section{ФУНКЩЇ ЛАІРАНЖА Ӥ ГАМІЛЬТОНА РЕЛЯТИВІСТСЬКОЇ СИСТЕМИ ЗАРЯДЖЕНИХ ЧАСТИНОК}

Л. Ф. Блажиєвський, Б. В. Будний, Ю. С. Криницький

Львівський національний університет імені Івана Франка, кафедра теоретичной фізики,

вул. Драгоманова, 12, Львів, 79005, Украӥна

Розглянуто релятивістське узагальнення функџій Лагранжа й Гамільтона для класичної системи зарятжених частинок. Отримано локальний вираз для лагранжіяна в першому наближенні за константою взаємодії $e^{2}$. Показано, що цей результат неоднозначний. Ця неоднозначність, однак, не проявляється в розглянутому наближенні. Після застосування додаткових наближень знайдено релятивістську функцію Гамільтона з екранованою взаємодією. 